\title{
On the Gain and Link Equation for Reactive Millimetre-wave Surface Wave Propagation System
}

\author{
Kin-Fai Tong \\ Department of Electronic \& Electrical Engineering, \\ University College London \\ Torrington Place, London, WC1E 7JE, United Kingdom \\ k.tong@ucl.ac.uk
}

\author{
JiXiang Wan, XuDong Wang ChunBang Wu \\ Xi'an Institute of Space Radio Technology, \\ Xi'an, Shaanxi, 710100,China \\ 13992841372@163.com
}

\begin{abstract}
Surface-wave has been proven to be the third option between wired and wireless communication. It has been proposed to be utilised for inter-machine and on-body communications, because of its on-surface two-dimensional feature. It provides a flexible two-dimensional platform and shows many advantages such as power savings, excellent electromagnetic compatibility characteristic, non-line-of-sight communication over traditional space wave wireless communication systems. When compared to wired systems, surface wave systems can provide a wide band channel for one-to-many/many-to-one communications. This work we will demonstrate our recent work in the concept of surface wave gain and formulate the surface wave wireless link equation for the first time. A $52 \mathrm{GHz}$ 3-dB bandwidth test bed was constructed to validate the theoretical and simulation results in the proposed method and equation.
\end{abstract}

Keywords - Surface wave; reactive surface; link equation

\section{INTRODUCTION}

Surface wave (SW) first identified by Zenneck in 1907 [1] is the one that propagates along the interface between two media without radiation. A lot of theory and experiments about Zenneck SW, including several kinds of different reactive surfaces and transducers, especially on how to excite surface wave efficiently were reported between 1950s - 1970s [2]-[11]. However, the SW seems to be forgotten between the 1980s and 2000s. Recently, traditional wired communication systems and space wave wireless communication systems are facing bottlenecks in some applications such as ultra-wideband communication over the sea [12], wireless body area networks (WBANs) [13], millimeter-wave wireless interconnects for networks-on-chip [14]. SW has been theoretically demonstrated to have a high-throughput for inter-chip communication with lower power loss, and that it can be readily applied as inter-chip communication fabric [15],

As the extension of our work reported, [16], [17], in surfacewave excitation efficiency, the relationship between the SW excitation efficiencies, the aperture height, surface reactance and optimum surface reactance, and the directional propagation characteristic of an aperture transducer study, we will present our recent work in the concept of surface wave gain and the deviation of the surface wave wireless link equation for the first time. A $52 \mathrm{GHz} 3-\mathrm{dB}$ bandwidth test bed was constructed to validate the theoretical and simulation results in the proposed method and equations.

\section{THE SURFACE WAVE LINK EQUATION}

The link budget equations provide important information to evaluate the performance of a SW communication system. Although some work has been reported recently, a general equation is not available yet. In [14], the SW link power dissipation was calculated based on the transmission line model, and the parameters are extracted from the measured results in [13]. Hence the proposed model in [14] is only applicable to the specific surface wave communication system consisting of the particular transducers and surface reactance used in [13]. The concept of surface wave gain and the angular coverage characteristics were not considered. In this section, the link budget equation of the surface wave communication systems is introduced. The basic surface wave communications link, shown in Fig. 1, identifies the basic parameters of the link.

The parameters of the link are defined as: $P_{t}=$ input power (watts); $P_{r}=$ received power (watts); $G_{t}=$ transmitting transducer gain; $G_{r}=$ receiving transducer gain; and $R=$ separation between the transmitting and receiving transducers (meters).

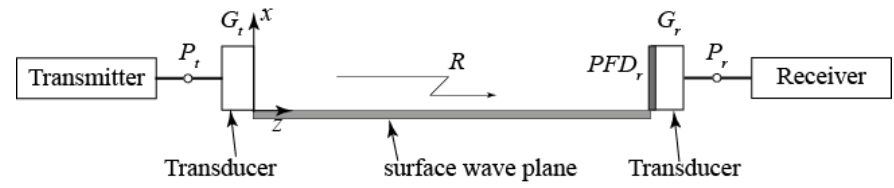

Fig. 1 Basic surface wave communication link

\section{A. The gain of a surface wave transducer}

As shown in Fig. 2, consider a surface wave propagating along a reactive surface from a point source $S$ of power $P_{t}$. The wave propagation is isotropic over the surface, i.e. radially propagation from the point source $S$. The normalized propagation pattern of an isotropic transducer is simply a circle of a unit radius in the $\phi$-direction; the total power is therefore $2 \pi$ for the complete surface. According to the law of conservation 
of energy, for the directivity function of any other transducer, the value of the integral is the same. Thus

$$
2 \int_{0}^{\phi_{0}}\left(E_{o} \cos \frac{\pi \phi}{2 \phi_{0}}\right)^{2} d \phi=2 \pi
$$

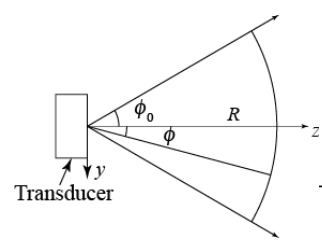

(a)

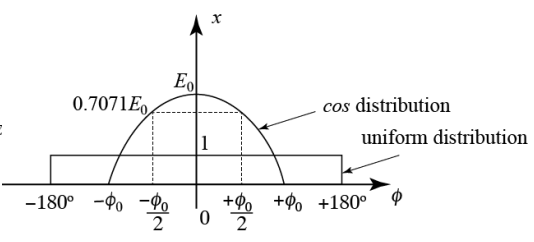

(b)
Fig. 2 (a) The SW angular coverage of a rectangle aperture transducer (top view), (b) Surface wave beam excited by a aperture with $c o s$ distribution and uniform distribution

The solution of Eq. 1 is:

$$
E_{0}=\sqrt{\frac{2 \pi}{\phi_{0}}}
$$

Similar to antenna directivity in space wave, the SW directivity can be defined as the ratio of the power intensity of the transducer in a particular direction and that of an isotropic source fed by the same amount of power. Since the directivity of an isotropic source is 1 , it is then obvious that the directivity of a directional transducer can be expressed as

$$
D=E_{0}^{2} \approx 5.14 \frac{W}{\lambda}=\eta_{a} \frac{2 \pi}{\lambda} W
$$

Where the factor $\frac{2 \pi}{\lambda} W$ represents the directivity of a uniformly illuminated constant phase aperture; $\eta_{\mathrm{a}}=81.8 \%$ is the aperture efficiency of the transducer defined as ratio of power loss due to non-uniform cosine amplitude distribution over the excitation aperture width.

Further, if all the losses are included, we can obtain the gain expressed as

$$
G=\eta \frac{2 \pi}{\lambda} W
$$

where $\eta$ is the total efficiency of the transducer and can be defined as

$$
\eta=\eta_{a} \eta_{s} \eta_{r} \eta_{m}
$$

where $\eta_{\mathrm{s}}$ is the surface wave excitation efficiency depending on the aperture height and surface reactance, $\eta_{\mathrm{r}}$ is the reflection (impedance mismatch) efficiency, $\eta_{\mathrm{m}}$ is the dielectric/conduction efficiency due to not ideal material.

It is then obvious that the surface wave gain is proportional to the aperture width $W / \lambda$. Note also that the effective aperture width can be expressed as

$$
A=\frac{G \lambda}{2 \pi \eta}
$$

Then, the scope of application of the cosine function model adopted in this paper will be discussed. Using Eq. 3, the surface wave directivity expressed in $\mathrm{dB}$, will be

$$
\begin{gathered}
G=20 \log \left(E_{0}\right)=10 \log (2 \pi)-10 \log \left(\phi_{0}\right) \\
=8.0-10 \log \left(\phi_{0}\right)
\end{gathered}
$$

When, we can find the minimum surface wave $G_{\min }$ :

$$
\mathrm{G}_{\min }=3 \mathrm{~dB}
$$

Using the equation

$$
\phi_{3 d B}=70 \frac{\lambda}{W}\left(^{\circ}\right)
$$

derived in [16], we can obtain

$$
\phi_{\max }=70 \frac{\lambda}{D_{\min }}\left({ }^{\circ}\right)=180^{\circ}
$$

Hence, the minimum aperture width corresponding to the minimum surface wave gain can be found is

$$
D_{\min }=0.39 \lambda
$$

If the width is less than $D_{\text {min }}$, the proposed model will be invalid. In fact, the transducer with the width less than $0.6 \lambda_{0}$ is not desirable because of the poor impedance matching.

\section{B. Power Flux Density}

The power flux density $P F D_{\mathrm{r}}$ (please refer to Fig. 1), usually expressed in $\mathrm{Wm}^{-2}$, at the distance $R$ from the transmitting transducer with a transducer gain $G_{t}$, is defined as

$$
P F D_{r}=\frac{P_{t}}{2 \pi R} G_{t} \quad\left(\mathrm{Wm}^{-2}\right)
$$

This relationship demonstrates that the power density of a surface wave propagating from a source is inversely proportional to the distance from the source not to the wellknown inverse square law of space wave radiation.

\section{Basic Link Equation for Received Power}

We now have all the elements necessary to define the basic link equation for determining the received power at the receiver terminals for a surface wave communications link. Consider now a receiver with the gain $G_{r}$ located a distance $R$ from a transmitter of $P_{t}$ watts and transducer gain $G_{t}$, as shown in Fig. 1 The power $P_{r}$ intercepted by the receiving transducer will be

$$
P_{r}=P F D_{r} W_{e}
$$

Substituting Eq. 6 into Eq. 12 and Eq. 13 yields

$$
P_{r}=\frac{P_{t} G_{t}}{2 \pi R} \frac{G_{r} \lambda}{2 \pi}
$$

Rearranging Eq. 14 into 


$$
\frac{P_{r}}{P_{t}}=\frac{G_{t} G_{r}}{(2 \pi)^{2}} \frac{\lambda}{R}
$$

Substituting Eq. 4 into Eq. 15

$$
\frac{P_{r}}{P_{t}}=\eta_{t} \eta_{r} \frac{W_{t} W_{r}}{R \lambda}
$$

Further, if some losses $\mathrm{L}_{\mathrm{s}}(\mathrm{dB})$ from not ideal reactive surface is also considered, (28) can be written as in $\mathrm{dB}$

$$
S_{21}=10 \log \left(\frac{P_{r}}{P_{t}}\right)-L_{s}=10 \log \left(\eta_{t} \eta_{r} \frac{W_{t} W_{r}}{R \lambda}\right)-L_{s}
$$

This result gives the basic link equation, sometimes referred to as the link power budget equation, for a surface wave wireless communications link.

\section{Experimental setup}

To verify the proposed theoretical study, an experiment setup as shown in Fig. 3 was built. Two SW transducers based on the theoretical results centered at $60 \mathrm{GHz}$ for covering the millimeter-wave frequency range are designed. The separation between the two transducers was fixed at $140 \mathrm{~mm}\left(28 \lambda_{0}\right.$ at 60 $\mathrm{GHz}$ ) for the measurement. A Keysight N5250A-017 millimeter-wave network analyzer was used for the transmission (S21) test of the surface wave platform. The network analyzer was first calibrated and normalized to eliminate the cable effect and the impedance mismatch of SW transducers, i.e. $\mathrm{S}_{21}$ equals $0 \mathrm{~dB}$ when the two transducers were directly connected. Then the two transducers are placed on a piece of conventional microwave substrate. Taconic TLY -5 microwave substrate $\left(\varepsilon_{r}=\right.$ 2.2 , thickness $=0.38 \mathrm{~mm}$, loss tangent at $10 \mathrm{GHz}=0.0009,300$ $\times 300 \mathrm{~mm}^{2}$ ) was selected based on the surface impedance $\left(X_{s}\right)$ it can provide and the low dielectric loss when compared to other microwave substrates available in the university. The surface impedance of the Taconic TLY-5 substrate at $60 \mathrm{GHz}$ is $j 100 \Omega$ which was evaluated by using Eq. 3 and Eq. 4 described in [7].

$$
X_{s}=2 \pi f \mu_{0}\left[\frac{\varepsilon_{r}-1}{\varepsilon_{r}} h_{s u b}+\frac{\Delta}{2}\right]
$$

where the skin depth of a metal sheet, $\Delta$, is

$$
\Delta=\sqrt{\frac{1}{\pi f \mu_{0} \sigma}}
$$

and the thickness of the dielectric layer, $h_{s u b}$, its dielectric constant, $\varepsilon_{r}$, the frequency of operation, $f$, and conductivity $\sigma$.

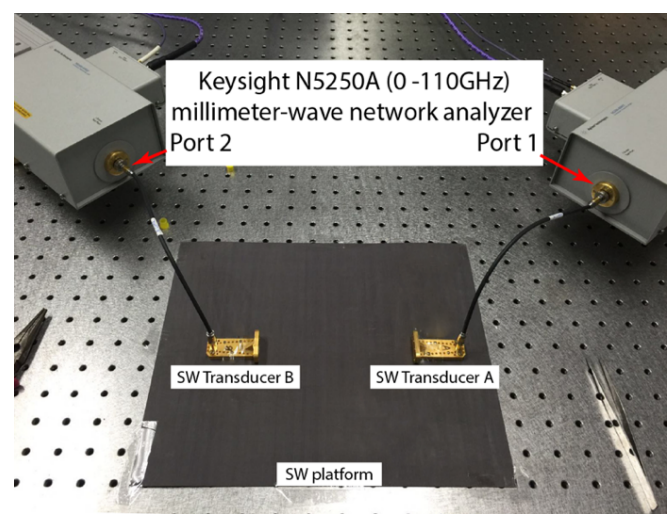

Fig. 3 The measurement setup of the propagation loss (S21)

\section{E. Experimental Results}

The measured transmission on the SW platform is shown in Fig. 3. It can be observed that the $3 \mathrm{~dB}$-transmission-bandwidth is about $52 \mathrm{GHz}$ from 34.4 to $86.4 \mathrm{GHz}$. Theoretically, such SW platform can support communication systems require data rate in the order of tens of Gbps. Moreover, the average transmission over the $140 \mathrm{~mm}$ separation is about $-16 \mathrm{~dB}$, which is much higher than that of the free space communication. The measured results generally follow the trend of the simulated results, both results match well with each other below $45 \mathrm{GHz}$, and the discrepancy starts to increase to about $1 \mathrm{~dB}$ up to $60 \mathrm{GHz}$, but it increases further to $3 \mathrm{~dB}$ after $60 \mathrm{GHz}$. The authors suspect such difference may cause by the relative higher dielectric loss at higher frequency in the microwave substrate. As explained, the surface impedance of the presented model is only $j 100 \Omega$, rather than the optimal $j 250 \Omega$ reported in [16], it is also expected that the transmission will be higher if the SW platform is realized by a proper microwave substrate, although the transmission bandwidth will be of similar order.

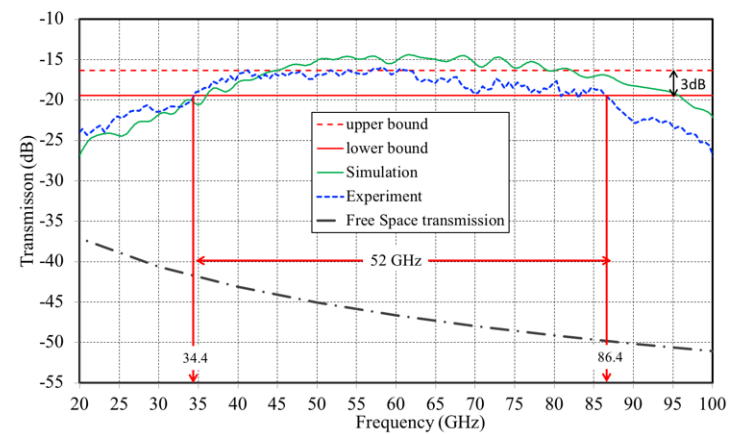

Fig. 4 Comparison of the simulated and measured S21

\section{CONCLUSION}

This paper extended our recent investigation in aperture transducers on a reactive surface to the concept of surface wave transducer gain and the surface wave wireless link equation systematically. The simulation results illustrate the validity of the proposed method and theory and verified by measurement results. Besides, a prototype of a $52 \mathrm{GHz}$ wide $3 \mathrm{~dB} \quad \mathrm{~S}_{21}$ bandwidth surface wave wireless communication system has also implemented to demonstrate the advantages of the surface wave technologies.

The surface wireless communication systems show many advantages, such as one-to-many (1-to-M) communication, power savings, excellent EMC characteristic, non-line-of-sight communication, will receive more extensive attentions in the future. This work can support the designs of the surface wireless communication systems including a transducer, reactive surface and link system performances and will furtherly speed up the application of the surface wireless communication systems.

\section{ACKNOWLEDGMENT}

The academic visit of Dr. Wan at UCL was co-sponsored by the China Scholarship Council and Xi'an Institute of Space Radio Technology. We would also like to express our appreciations to Dr. Kungbo Ng from the State Key Laboratory of Millimeter Waves at the City University of Hong Kong for his support in the experimental work. 


\section{REFERENCES}

[1] J. Zenneck, "Propagation of plane EM waves along a plane conducting surface," Ann. Phys., vol. 23, pp. 846-866, 1907.

[2] G. Rich, "The launching of a plane surface wave," Proc. IEE-Part B Radio Electron. ..., no. 1783, 1955.

[3] W. . M. . G. . FERNANDO and H. . E. . M. . BARLOW, "an Investigation of the Properties of Radial Cylindrical Surface Waves Launched Over Flat Reactive Surfaces,” pp. 307-318, 1956.

[4] B. K. P. Sharma, M. Sc, and D. Ph, "An Investigation of the Excitation of Radiation by Surface Waves," no. 2737, pp. 116-122, 1959.

[5] B. K. P. Sharma, "The Estimation of the Reactance of a Loss-free Surface Supporting Surface Waves,” no. 2881, 1959.

[6] B. P. A. L. Cullen, D. Ph, J. A. Staniforth, and D. Ph, "the Launching of Surface Waves By an End-Fire Array of Slots," vol. 0, no. 450, pp. 492-495, 1961.

[7] H. M. Barlow and J. Brown, "Radio Surface Waves," Oxford University Press, 1962.

[8] E. L. Johansen, "Surface Wave Scattering by a Step," IEEE Trans. Antennas Propag., vol. 15, no. 3, pp. 442-448, 1967.

[9] D. Hemmendinger and F. J. Zucker, "A Forgotten Theorem and its Application to Surface-Wave Excitation," IEEE Trans. Antennas Propag., vol. 18, no. 1, pp. 132-133, 1970.

[10] D. A. Hill and J. R. Wait, "Excitation of the Zenneck surface wave by a vertical aperture," Radio Sci., vol. 13, no. 6, pp. 969-977, 1978
[11] G. L. James, "Surface Reactance of Corrugated Planes," Electron. Lett., vol. 15, no. 23, pp. 751-753, 1979.

[12] J. LaComb and P. Mileski, "Ultra Wideband Surface Wave Communication,” Prog. Electromagn., vol. 8, pp. 95-105, 2009.

[13] J. E. Turner, M. S. Jessup, and K. F. Tong, "A novel technique enabling the realisation of $60 \mathrm{GHz}$ body area networks," Proc. BSN 2012 9th Int. Work. Wearable Implant. Body Sens. Networks, no. 1, pp. 58-62, 2012.

[14] A. J. Karkar, J. E. Turner, K. Tong, R. Al-Dujaily, T. Mak, A. Yakovlev, and F. Xia, "Hybrid wire-surface wave interconnects for next-generation networks-on-chip," IET Comput. Digit. Tech., vol. 7, no. 6, pp. 294-303, 2013.

[15] A. Karkar, T. Mak, K. F. Tong, and A. Yakovlev, "A Survey of Emerging Interconnects for On-Chip Efficient Multicast and Broadcast in Many-Cores," IEEE Circuits Syst. Mag., vol. 16, no. 1, pp. 58-72, 2016.

[16] J. Wan, K. F. Tong, and C. Wu, "The excitation efficiency of surface waves on a reactive surface by a finite vertical aperture," in 2015 IEEE International Symposium on Antennas and Propagation \& USNC/URSI National Radio Science Meeting, 2015, pp. 16341635 .

[17] J. Wan and K. Tong, "Simulation and Experimental Verification for a $52 \mathrm{GHz}$ Wideband Reactive Surface Wave Propagation System," IEEE Trans. Antennas Propag. 